\title{
ANALYSING APPLICABILITY OF SELECTED METHODS TO SMOOTH INDICATOR DIAGRAMS OF MARINE MEDIUM-SPEED ENGINE
}

\author{
Rafał Pawletko, Ph. D. \\ Gdynia Maritime University, Poland
}

\begin{abstract}
The article analyses the applicability of selected smoothing methods to smooth indicator diagram curves and to filter disturbances. An intermediate goal of the study was an attempt to extract disturbances recorded during pressure curve smoothing, which are believed to be a source of important diagnostic information.

Within the framework of the reported analysis, a comparison was made between the moving average method, the Savitzky-Golay filter, and the frequency filtration method. The research was performed on a marine medium-speed engine Sulzer $3 \mathrm{Al}$ 25/30, which has a relatively long indicator passage.
\end{abstract}

Keywords: indicator diagrams, smoothing waveforms, filtering disturbances

\section{Introduction}

Measuring the pressure inside the cylinders of marine piston engines is an important issue when analysing both the combustion course, and the technical state of the engine. A basic area in which the information concerning the cylinder pressure is used refers to the assessment of heat emission characteristics based on the first law of thermodynamics $[4,6,7]$. These characteristics can then make a basis for: verifying the prepared combustion process simulation models, developing new concepts of combustion systems, analysing the behaviour of alternative fuels and fuel injection systems, etc.

Reliable information on the combustion process can be used for on-line control of engine operation. Controlling the course of combustion may also result in limiting the emission of toxic fuel components. For instance, contemporary electronically controlled marine engines can operate in a so called Low NOX mode (economical mode with limited emission of toxic compounds).

A condition to obtain reliable heat emission characteristics is the smooth and disturbance-free pressure curve. Meeting this condition in practice is extremely difficult. A number of important factors concerning cylinder pressure measurements can be named which remarkably deform the recorded pressure time-histories:
- the pressure sensor is situated on the indicator valve and not directly inside the combustion chamber;

- the indicator valve is connected with the combustion chamber through the indicator passage, the length and volume of which can be relatively large, depending on the engine structure;

- $\quad$ pressure measurements making use of the indicator passage lead to phase and amplitude disturbances of the recorded signal, and introduce oscillating disturbances generated by vibrations of the gas column inside the passage;

- measurements performed in changing pressure and high temperature conditions place very high demands on the used sensors in terms of their accuracy and reliability;

- $\quad$ the used measuring systems are susceptible to electric disturbances generated both by engine subsystems, and other engine power plant units which emit the electromagnetic field.

A reliable and disturbance-free pressure curve makes a basis for further analysed of the combustion process. Despite remarkable development in production of pressure sensors and measuring systems, a problem which still needs solving concerns postprocessing of the already recorded timehistories. An obligatory procedure in this situation is averaging pressure curves recorded in a number of measuring cycles. 
Depending on the adopted system, this averaging can include from ten to several tens of realisations. In most cases, however, averaging is not sufficient, especially in analyses of heat emission characteristics, and complementary numerical data filtering or smoothing turns out necessary.

The article analyses the applicability of selected smoothing methods to smooth the indicator diagram curves, with an intermediate attempt to extract the disturbances recorded during pressure curve smoothing. These disturbances are believed to be a source of important diagnostic information.

\section{Types and sources of disturbances in indicator diagram measurements}

Disturbances affecting the waveform of the indicator diagram curve can be divided into two basic groups. The first group refers to the nature of pressure measurements in marine engine cylinders, in particular to the presence of the indicator passage. The second group includes disturbances of electrical origin, generated by engine systems and auxiliary devices, and recorded by the measuring lines. The latter type of disturbances gains in importance in the marine power plant environment [5].

Pressure sensors are mounted on the indicator valve, and not directly inside the engine cylinder. Depending on the engine structure, the length of the indicator passage linking the indicator valve with the combustion chamber can reach one meter. Such a passage, of a relatively large length and small diameter, is a possible source of both absolute pressure estimation errors, and phase errors resulting from the fact that the pressure wave covers the distance between the combustion chamber and the measuring sensor. According to [3], the geometry of the indicator passage and the rotational speed of the engine affect remarkably the waveform of the indicator curve. The maximal pressure estimation error increases with the increasing length of the indicator passage and with the increasing rotational speed of the engine, and decreases with the increasing diameter of the indicator passage. No effect of the engine load on the pressure estimation error has been detected [3].

The examination reported in [2] has revealed strong relation between the diameter of the indicator passage and that of the sensor. It was demonstrated that when these two diameters are close to each other, this may enable to eliminate resonance frequencies and record a curve which is free of oscillating disturbances.

The effect of the passage which connects the combustion chamber with the indicator sensor on the waveform of the measured curves has also been confirmed in [2]. There, a proposal was made to modify the structure of the indicator passage and to install an additional indicator sensor adapter to eliminate resonance vibrations.

The problem of disturbances introduced by indicator passages is of high importance, which has been confirmed by results of other analyses in this area. However, despite high theoretical potential for introducing structural modifications to the indicator passage in order to minimise the measuring errors, especially in the resonance frequency ranges, this approach is unlikely to be widely used in practice. An alternative for structural modifications is an attempt to smooth the recorded curves and/or to filter the disturbances during data postprocessing [2].

\section{Data smoothing methods used in indicator diagram postprocessing}

A basic method of indicator diagram smoothing, which is in a wide use nowadays, is averaging a number of curves recorded in stationary conditions. This averaging, which can include from ten to several tens of realisations, enables to eliminate, first of all, random disturbances recorded in single realisations.

The most popular method to smooth the measured data is the moving average method, which consists in replacing each value in the data set by the value averaged over the interval of a given width. This procedure is equivalent to a low-passing filter with the response given by the following equation:

$$
y_{s}(i)=\frac{1}{2 N+1}(y(i+N)+y(i+N-1)+\cdots+y(i-N)
$$

where:

$\mathrm{y}_{\mathrm{s}}(\mathrm{i})$ - smoothed value at $\mathrm{i}$-th point,

$\mathrm{N}$ - number of points taking part in averaging on each side of the central point $\mathrm{y}_{\mathrm{s}}(\mathrm{i})$,

$2 \mathrm{~N}+1$ - width of the moving interval of averaging.

A generalisation of the method making use of the moving average is the Savitzky-Golay filter [8]. Filter coefficients are calculated by approximating the real curve with a polynomial of a given order, using the least-squares method. Like for the moving average method, the approximation is conducted within the moving interval of a given width, and that is why the Savitzky-Golay filter bears the name of the moving polynomial approximation. The use of a polynomial of a sufficiently high order enables to obtain high smoothing efficiency without losing essential information carried by the original signal. The Savitzky-Golay filter is very efficient in terms of preservation of high-frequency signal components, but can be less efficient, as compared to the moving average method, in eliminating random noise.

While the moving approximation can be considered natural extension of the moving average concept, the use of multiple moving approximation to process the measured data should be considered an achievement of utmost importance. The moving approximation consist in calculating the approximated value at one point within the approximation interval. This interval moves along the measuring axis, or is on-line created when new measuring data are delivered to the analysing systems. The data set which is used as an approximation basis for a subsequent step (passing), contains the results of approximation from the previous approximation step. In each step, new sets of coefficients are calculated for the approximating formula.

Disturbances can also be filtered out from the indicator diagrams using directly numerical filters. Taking into account 
the nature of these disturbances, of highest applicability in this situation will be the low-pass filters with limited impulse response due to the linear nature of the phase shift [1].

\section{Research rig}

The experiment was performed on the research rig the main element of which was the marine four-stroke engine Sulzer $3 A 125 / 30$. Basic technical specifications of the engine are the following:

- product designation - $3 \mathrm{Al}$ 25/30

- number of cylinders - 3

- cylinder diameter [mm] - 250

- piston stroke [mm] - 300

- piston displacement [cm3] - 14726

- nominal power $[\mathrm{kW}]-408$

- rotational speed [rpm] - 750

- compression ratio -13

Al 25/30 type engines are used as main propulsion on small ships and for driving electric current generators in ship power plants.

Indication was done using the electronic indicator Unitest 201, the angular resolution of which is $0,5^{\circ} \mathrm{OWK}$. The measurements were performed using 6353A24 sensors made by Kistler. All measurements were done for the engine rotational speed of $750 \mathrm{rpm}$.

The gas passage connecting the indicator with the combustion chamber in this engine is relatively long and has a complicated shape (Fig. 1).

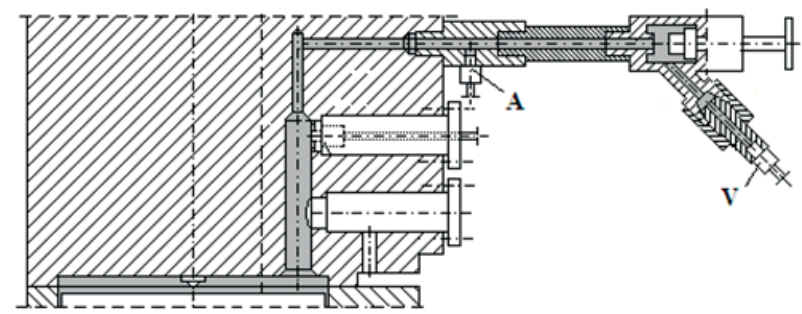

Fig. 1. Gas passage and indicator valve structure, with the distribution of combustion pressure sensors within the cylinder space of the examined engine A25: A - pressure sensor and measuring point with 7523A10 adapter made by Kistler, $V$-indicator valve

\section{Smoothing indicator diagrams of the laboratory engine Al 25/30}

The indicator diagram of the engine $\mathrm{Al} 25 / 30$ is a pressure curve with disturbances of various nature. Al 25/30 is a medium-speed engine with a relatively long and complicated indicator passage. A sample indicator curve for this engine is shown in Fig. 2. The curve was obtained as an average of 16 realisations.

\section{Curve smoothing using the moving average method}

The here reported research started with and attempt to smooth the indicator pressure curve using the moving average method. First, single smoothing was applied with the moving interval consisting of $\mathrm{k}=20$ points. The original and smoothed waveforms are shown in Fig. 3, while their derivatives are presented in Fig. 4.

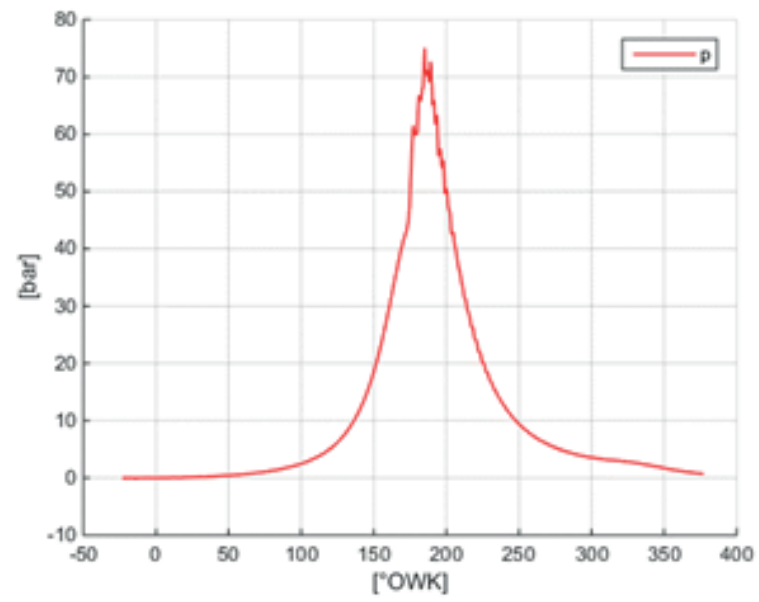

Fig. 2. Indicator diagram of the engine Al 25/30

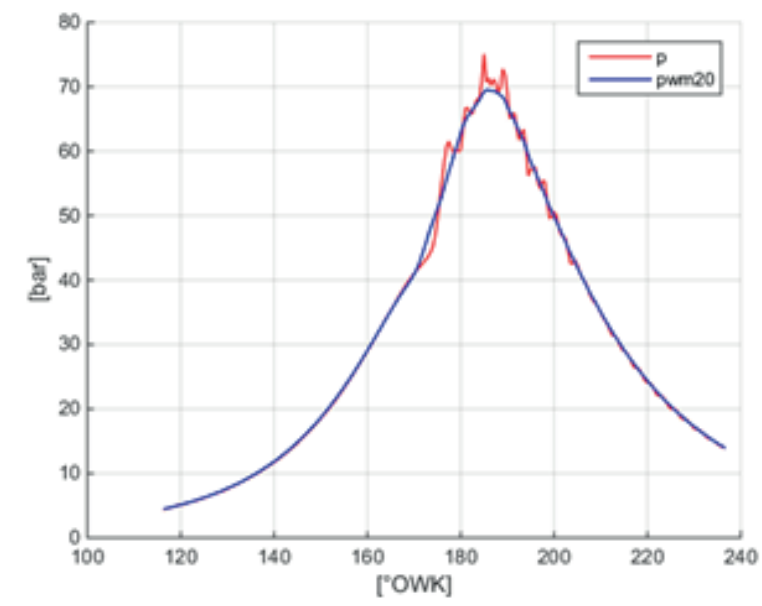

Fig. 3. Indicator curve smoothed using the moving average method with the moving interval consisting of $k=20$ points ( $p w m 20$ ), as compared to the original curve $(p)$

Despite the use of the moving interval consisting of 20 points, the obtained waveform of the derivative dpwm 20 is not sufficiently smooth. An additional limitation of the moving average method is that it introduces some phase error, which can be observed on the smoothed derivative, when compared to that of the unsmoothed pressure curve. The theoretical start of the combustion is at about $172^{\circ} \mathrm{OWK}$, while the angle value determined from the smoothed derivative is situated at about $169^{\circ} \mathrm{OWK}$. The phase error in this case is approximately equal to $4^{\circ} \mathrm{OWK}$. Increasing the number of points composing the averaging interval (interval width) above 20 does not lead to the improvement of smoothing quality, and at the same time introduces further phase and amplitude errors.

Better results of smoothing can be obtained using so-called multiple smoothing, proposed in [5]. The method consists in 
repeatable application of the smoothing procedure, with the same or similar parameters, to the already smoothed curve. This approach makes it possible to use a narrower smoothing interval. Figure 5 shows the results of smoothing after one (pwm1) and four successive repetitions of the smoothing procedure (pwm4). In each time the smoothing interval consisted of $\mathrm{k}=5$ points.

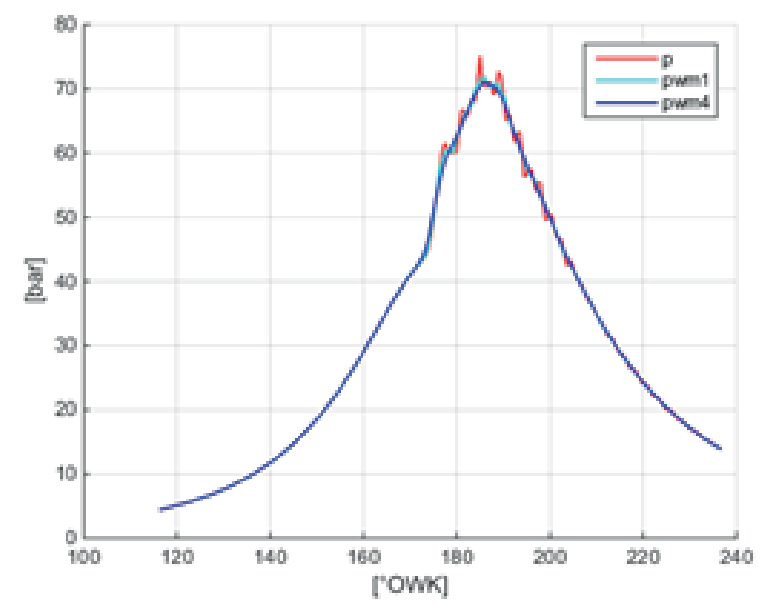

Fig. 5. Indicator curve smoothed using the moving average method with the moving interval consisting of 5 points, after single smoothing (pwm1) and four repetitions of smoothing ( $p w m 4$ )

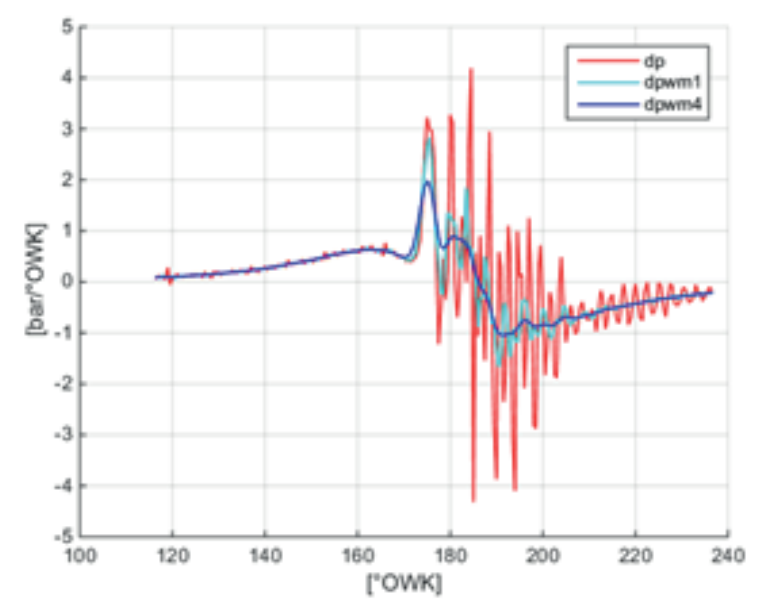

Fig. 6. Derivatives of the original curve $(d p)$ and the smoothed curves (dpwm1) and (dpwm4)

Four repetitions of the smoothing procedure enabled to filter effectively the disturbances, see Fig. 6, without introducing visible deformations and phase errors. The smoothed waveform is close to the original one, especially in the vicinity of the beginning of the combustion when the pressure increases dramatically. Multiple smoothing enabled to obtain the derivative waveform which is acceptable in terms of disturbances and free of significant phase and amplitude errors.

\section{Curve smoothing using the Savitzky-Golay filter}

The results of fourfold smoothing with the aid of the moving average method (pwm4) were compared with those obtained using the Savitzky-Golay filter (pwg4). In the latter case, use was made of the 3 rd order polynomial, and the width of the interval was equal to 12 points. The smoothing was repeated four times. The waveforms obtained using these two methods are compared, along with the corresponding derivatives, in Figs. 7 and 8.

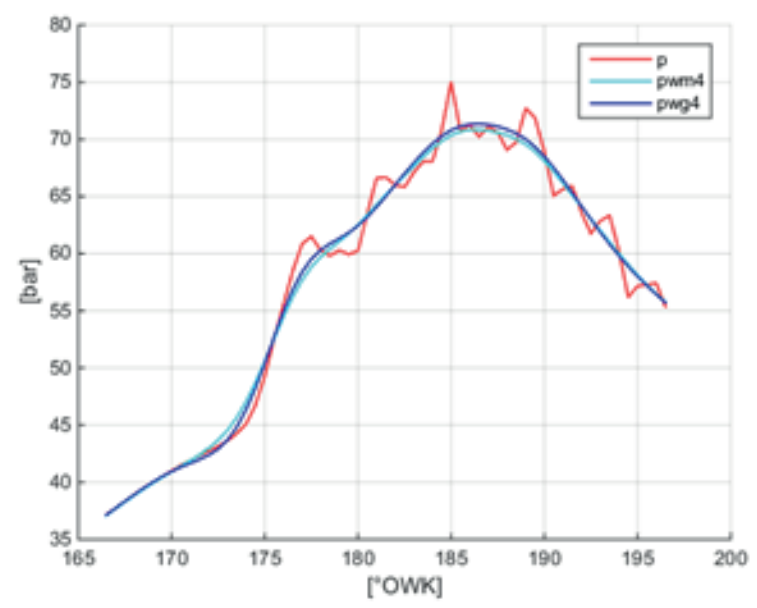

Fig. 7. Indicator curve smoothed using the moving average method with the interval consisting of $\mathrm{k}=5$ points ( $\mathrm{pwm} 4$ ), and the Savitzky-Golay filter with the interval consisting of $\mathrm{k}=12$ points (pwg4). In both cases smoothing was repeated four times.

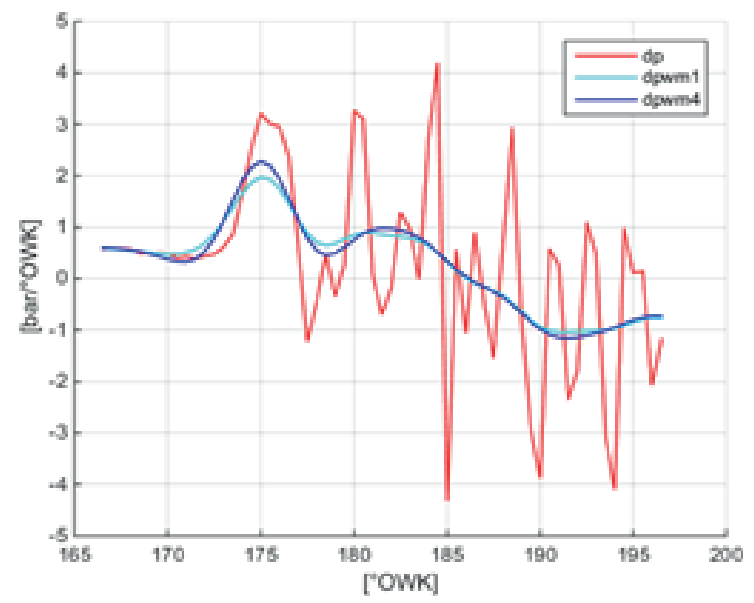

Fig. 8. Derivatives dpwm4 and dpwg4 of smoothed curves pwm4 and pwg4

Fourfold applications of the smoothing procedures based on the above two methods have returned similar results. Better robustness of the Savitzky-Golay filter can be observed in the areas of rapid curve changes, for instance at the beginning of the combustion. The curve smoothed using the filter is 
closer to the original shape, and introduces smaller phase and amplitude errors. This effect is especially noticeable on the waveforms of the calculated derivatives.

\section{Curve smoothing using the dedicated low-pass filter}

Analyses of indicator diagram postprocessing frequently indicate low efficiency of filtering based on the moving average methods [1], in particular in the frequency domain. As a consequence, an attempt was made to use a low-pass filter for smoothing purposes. Since the overwhelming majority of disturbances is situated in the upper part of the frequency range, the use of this filter is expected to effectively eliminate disturbances without losing important data within the frequency range of interest.

An essential issue in designing the low-pass filter is selecting the cut-off frequency. Its value depends on the nature of the observed disturbances and the engine rotational speed. The cut-off frequency should be selected individually for a given engine, which is a considerable limitation of the method.

In the here reported case, before selecting the cut-off frequency the power spectral density was prepared for the examined indicator curve to evaluate signal energy distribution in particular frequency bands. The power spectral density of this curve is shown in Fig. 9.

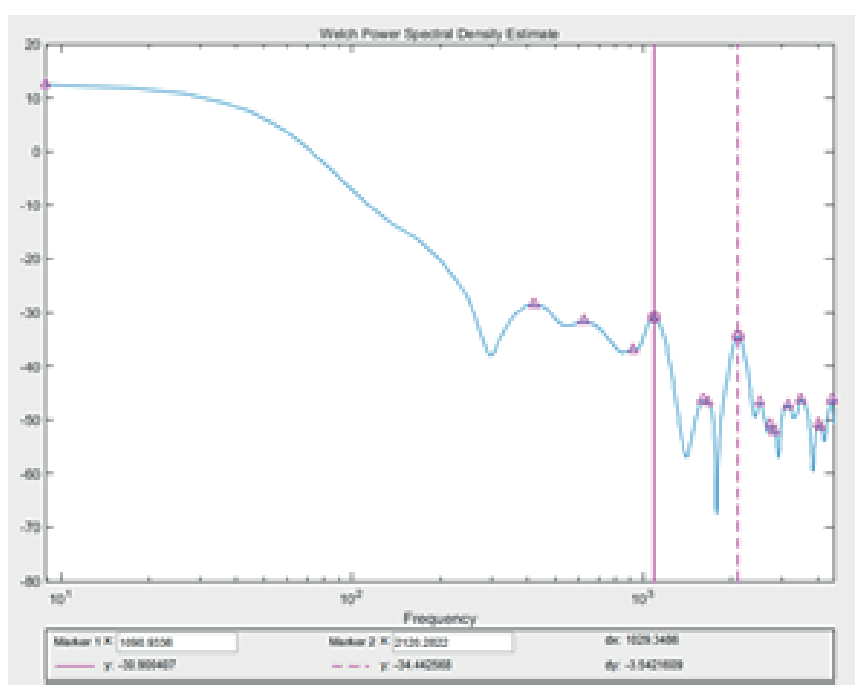

Fig. 9. Power spectral density of the pressure signal

The power spectral density curve reveals a decreasing trend for the increasing frequency, the local minimum of which is situated close to $1,8 \mathrm{kHz}$. Moreover, two local maxima can be observed at frequencies approximately equal to $1,1 \mathrm{k} \mathrm{Hz}$ and $2,1 \mathrm{kHz}$. Their presence may be connected with the disturbances introduced by the indicator passage.

Based on the signal spectrum curve a decision was made to assume the cut-off frequency for the designed filter at the level of $1 \mathrm{kHz}$. The designed filter is the 20th order low-pass filter with limited impulse response and Kaiser window. The effects of the use of this filter are shown in Figs. 10 and 11.

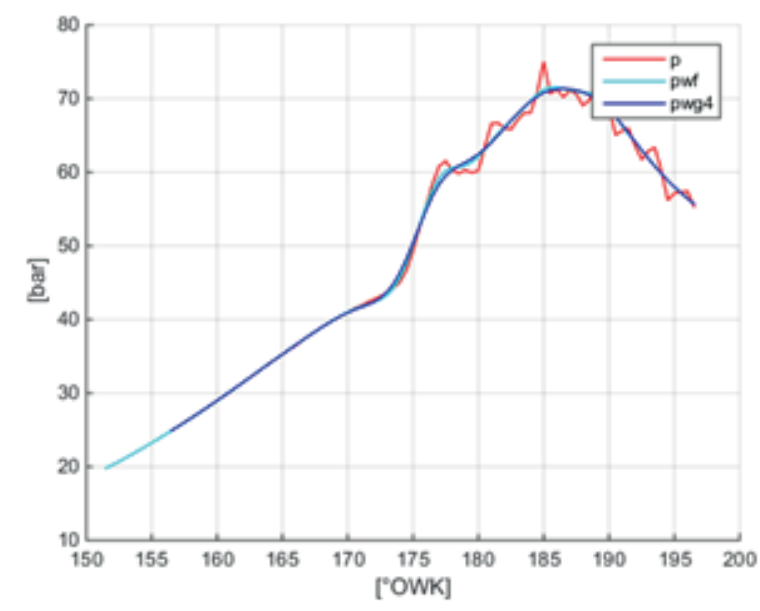

Fig. 10. Indicator curve smoothed using the low-pass filter with cut-off frequency of $1 \mathrm{kHz}(p w f)$, as compared to the curve smoothed using the Savitzky-Golay filter ( $p w g 4$ )

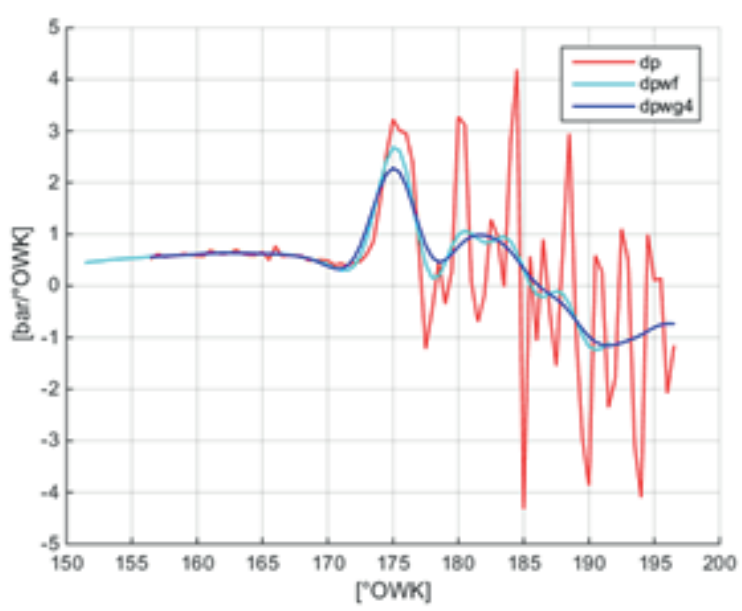

Fig. 11. Derivatives dpwf and dpwg4 of the smoothed curves pwf and pwg4

The use of the low-pass filter with the cut-off frequency of $1 \mathrm{kHz}$ enabled to obtain a curve which is very similar to that smoothed by multiple application of the Savitzky-Golay filter. However, its derivative, dpwf in Fig. 11, reveals a number of small waves. Decreasing the cut-off frequency to $800 \mathrm{~Hz}$ has made it possible to eliminate these waves and to obtain the analogous curve to that representing the Savitzky-Golay filter, see Fig. 12.

\section{Analysing disturbances of laboratory engine Al 25/30 indicator diagrams}

For each successive smoothing step, the smoothing deviation (disturbance) can be calculated as:

$$
d i f_{l-1 ; l}=d i f_{l-1}-d i f_{l}
$$

where:

$d i f_{l-1 ; l}$ - disturbance extracted for the given smoothing 


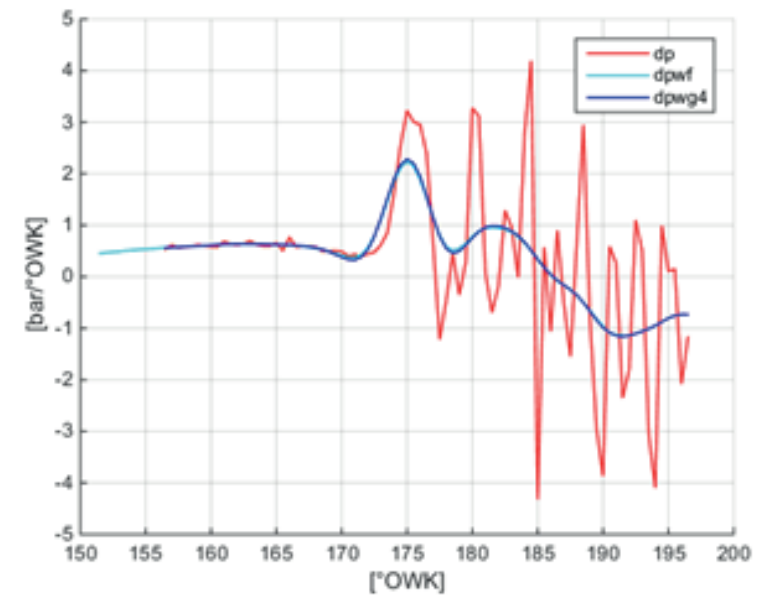

Fig. 12. Derivatives of curves smoothed using the low-pass filter with cut-off frequency of $800 \mathrm{~Hz}(d p f)$ and the Savitzky-Golay filter (dpwg4)

Figure 13 shows a set of disturbances \{dif1,dif2,dif3,dif4\} extracted in four successive steps of $\mathrm{p}$ curve smoothing, performed with the aid of the Savitzky-Golay filter. The applied filter made use of the 3rd order polynomial and the interval consisting of $\mathrm{k}=12$ points.

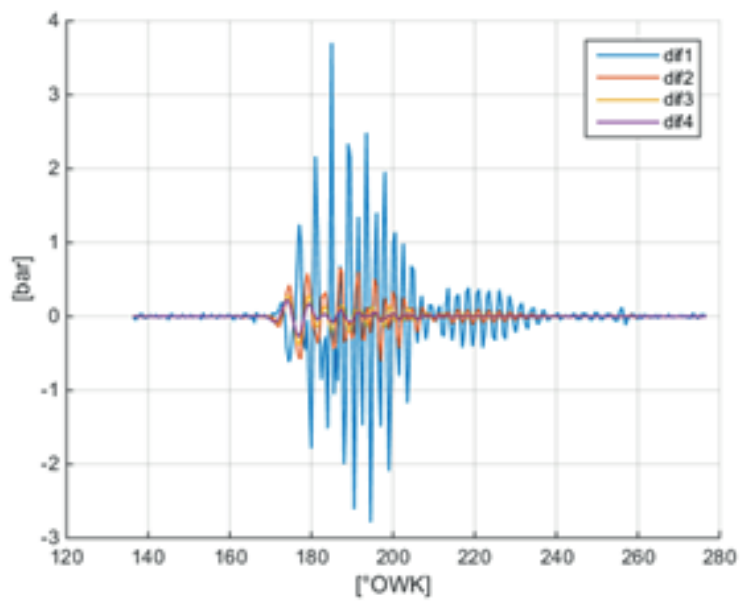

Fig. 13. Disturbances extracted in four successive steps of p curve approximation making use of the 3 rd order polynomial with $K=12$ (Fig. 1). Angle interval from 180 to $240^{\circ} \mathrm{OWK}$

Bering in mind processes which generate the curve p being the object of smoothing, it can be easily noticed that the extracted disturbances (Fig. 13) are mainly caused by gas oscillations initiated in the short measuring passage behind the combustion chamber by rapid start of the combustion process.

Figure 14 shows similar disturbance curves extracted for the angle interval between 70 and $140^{\circ} \mathrm{OWK}$, i.e. before the start of the combustion process.

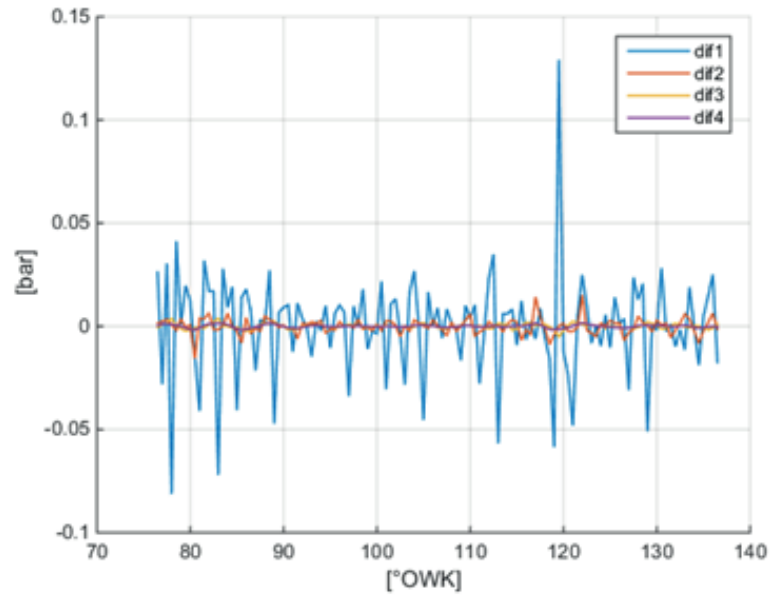

Fig. 14. Disturbances extracted in four successive steps of p curve approximation making use of the 3 rd order polynomial with $z K=16$ (Fig.1). Angle interval from 80 to $140^{\circ} \mathrm{OWK}$

The amplitude of the disturbances extracted before the start of the combustion process is lower by one order of magnitude, but their frequencies in both intervals are similar. Figures 15 and 16 show the disturbance signal frequency spectra for these two angle intervals.

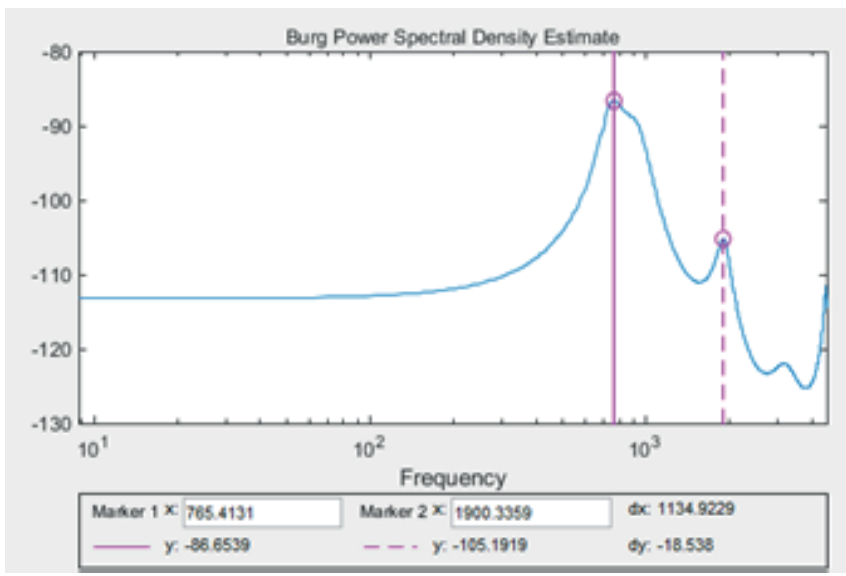

Fig. 15. Amplitude spectrum of disturbances extracted after step 1 of smoothing. Angle interval from 80 to $140^{\circ} \mathrm{OWK}$.

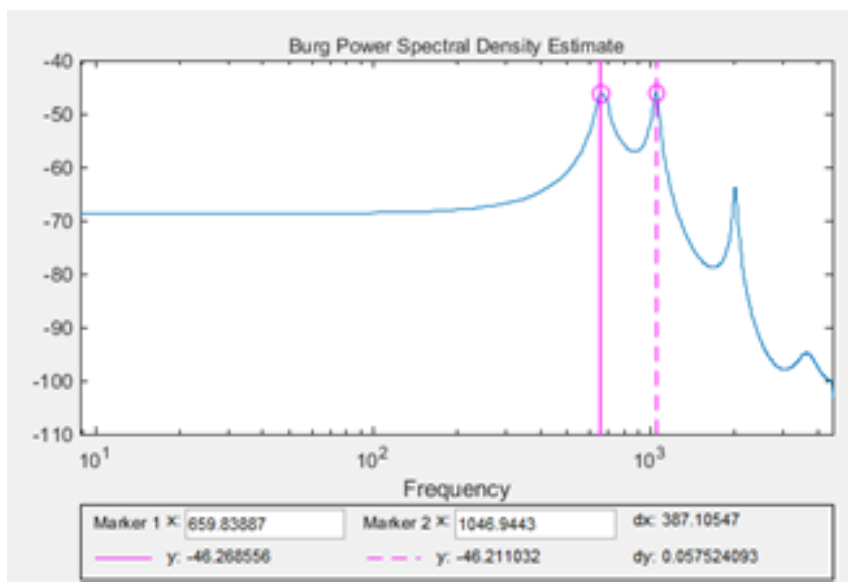

Fig. 16. Amplitude spectrum of disturbances extracted after step 1 of smoothing. Angle interval from 180 to $240^{\circ} \mathrm{OWK}$ 
The dominating frequency in the disturbance spectrum recorded before the start of the combustion process, the interval between $80^{\circ}$ and $140^{\circ} \mathrm{OWK}$ (Fig. 15), is $765 \mathrm{~Hz}$. Another local maximum which can be observed in this curve is situated at $1900 \mathrm{~Hz}$. In case of the spectrum recorded after the beginning of the combustion process, two clear maxima can be observed for the frequencies of $659 \mathrm{~Hz}$ and $1046 \mathrm{~Hz}$, accompanied by a lower local maximum at $2014 \mathrm{~Hz}$.

\section{Conclusions}

The examined smoothing methods applied to indicator diagrams of the medium-speed marine engine have made it possible to effectively filter out disturbances. However, the moving average method and the Savitzky-Golay filter need multiple smoothing with a relatively narrow moving interval to get satisfactory results. Single smoothing making use of a wide interval removes part of essential information from the original pressure curve.

The Savitzky-Golay filter was recognised as a better tool than the moving average method for smoothing rapid changes of the pressure curve, for instance the curve section corresponding to the beginning of the combustion process. The moving average method also introduces larger phase and amplitude errors.

High efficiency of curve filtering in the frequency domain has also been confirmed. The use of the low-pass filter with properly selected parameters enabled to obtain identical results as for the Savitzky-Golay filter.

Analysing the disturbances extracted from the indicator diagrams indicates that they are mainly caused by gas oscillations initiated in the short measuring passage behind the combustion chamber by rapid start of the combustion process.

\section{Bibliography}

1. Dey K., Characterization and rejection of noise from in-cylinder pressure traces in a diesel engine, Electronic Theses and Dissertations, Paper 121, 2012

2. Fujio N., Makato I., Errors of an Indicator due to a Connecting Passage, JSME 621.43.018.86

3. Hountalas D. T., Anestis A., Effect of Pressure Transducer Position on Measured Cylinder Pressure Diagram of High Speed Diesel Engines. Energy Convers, Mgmt Vol. 39, No. 7, pp 589-607, 1998

4. Pawletko, R., Polanowski, S, Influence of fuel injection system faults of marine diesel engine on the heat release characteristics, Combustion Engines, 154(3), 2013

5. Polanowski, S, Application of movable approximation and wavelet decomposition to smoothing-out procedure of ship engine indicator diagrams, Polish Maritime Research 2(52) Vol 14, pp. 12-17, 2007
6. Polanowski, S, Assessing diagnostic applicability of heat release characteristics determined based on ship engine indicator diagrams, Polish Maritime Research 3(61) Vol 16; pp. 32-35, 2009

7. Rychter, T., Teodorczyk, A., Mathematical modelling of piston engine operation cycle (in Polish), PWN, Warsaw 1990

8. Savitzky, A., Golay, M. J., Smoothing and differentiation of data by simplified least squares procedures, Analytical chemistry 36.8, 1964

9. Wlocardyk M.T., High accuracy glow-plug integrated cylinder pressure sensor for closed loop engine control, SAE Paper 2006-01-0184, 2006

\section{CONTACT WITH AUTHOR}

\author{
Rafał Pawletko \\ Gdynia Maritime University, \\ 81-87 Morska St. \\ 81-225 Gdynia \\ POLAND
}

